\title{
Lower Bounds Estimate for the Blow-Up Time of a Slow Diffusion Equation with Nonlocal Source and Inner Absorption
}

\author{
Zhong Bo Fang, Rui Yang, and Yan Chai \\ School of Mathematical Sciences, Ocean University of China, Qingdao 266100, China \\ Correspondence should be addressed to Zhong Bo Fang; fangzb7777@hotmail.com
}

Received 24 July 2013; Accepted 20 December 2013; Published 2 January 2014

Academic Editor: Mufid Abudiab

Copyright (C) 2014 Zhong Bo Fang et al. This is an open access article distributed under the Creative Commons Attribution License, which permits unrestricted use, distribution, and reproduction in any medium, provided the original work is properly cited.

We investigate a slow diffusion equation with nonlocal source and inner absorption subject to homogeneous Dirichlet boundary condition or homogeneous Neumann boundary condition. Based on an auxiliary function method and a differential inequality technique, lower bounds for the blow-up time are given if the blow-up occurs in finite time.

\section{Introduction}

Our main interest lies in the following slow diffusion equation with nonlocal source term and inner absorption term:

$$
\begin{gathered}
u_{t}=\Delta u^{m}+u^{p} \int_{\Omega} u^{q} d x-k u^{s}, \quad(x, t) \in \Omega \times\left(0, t^{*}\right), \\
u(x, 0)=u_{0}(x) \geq 0, \quad x \in \bar{\Omega},
\end{gathered}
$$

subject to homogeneous Dirichlet boundary condition

$$
u=0, \quad(x, t) \in \partial \Omega \times\left(0, t^{*}\right),
$$

or homogeneous Neumann boundary condition

$$
\frac{\partial u}{\partial v}=0, \quad(x, t) \in \partial \Omega \times\left(0, t^{*}\right),
$$

where $\Omega \subset \mathbb{R}^{3}$ is a bounded domain with smooth boundary $\partial \Omega, \bar{\Omega}$ is the closure of $\Omega, m>1, p \geq 0, q>0, s>1, p+q>$ $\max \{m, s\}, k>0, v$ is the unit outer normal vector on $\partial \Omega$, and $t^{*}$ is the possible blow-up time. By the maximum principle, it follows that $u(x, t) \geq 0$ in the time interval of existence. In the present investigation we derive a lower bound for the blow-up time $t^{*}$ when $\Omega \subset \mathbb{R}^{3}$ for the solutions that blow up.

Equation (1) describes the slow diffusion of concentration of some Newtonian fluids through porous medium or the density of some biological species in many physical phenomena and biological species theories. It has been known that the nonlocal source term presents a more realistic model for population dynamics; see [1-3]. In the nonlinear diffusion theory, there exist obvious differences among the situations of slow $(m>1)$, fast $(0<m<1)$, and linear $(m=1)$ diffusions. For example, there is a finite speed propagation in the slow and linear diffusion situation, whereas an infinite speed propagation exists in the fast diffusion situation.

The bounds for the blow-up time of the blow-up solutions to nonlinear diffusion equations have been widely studied in recent years. Indeed, most of the works have dealt with the upper bounds for the blow-up time when blow-up occurs. For example, Levine [4] introduced the concavity method, Gao et al. [5] employed the way of combining an auxiliary function method and comparison method with upper-lower solutions method, and Wang et al. [6] used the regularization method and an auxiliary function method. However, the lower bounds for the blow-up time are more difficult in general. Recently, Payne and Schaefer in $[7,8]$ used a differential inequality technique and an auxiliary function method to obtain a lower bound on blow-up time for solution of the heat equation with local source term under boundary condition (3a) or (3b). Specially, Song [9] considered the lower bounds for the blow-up time of the blow-up solution to the nonlocal problem (1)-(2) when $m=1$ and $p=0$, subject to homogeneous boundary condition (3a) or (3b); for the case $k=0$, we refer to [10].

Motivated by the works above, we investigate the lower bounds for the blow-up time of the blow-up solutions to 
the nonlocal problem (1)-(2) with homogeneous boundary condition (3a) or (3b). Actually, it is well known that if $p+$ $q>\max \{m, s\}$ and the initial value is large enough, then the solutions of our problem blow up in a finite time; one can see [11]. Unfortunately, our results are restricted in $\mathbb{R}^{3}$ because of the best constant of a Sobolev type inequality (see [12]).

This paper is organized as follows. In Section 2, we establish problem (1)-(2) with homogeneous Dirichlet boundary condition (3a). Problem (1)-(2) with homogeneous Neumann boundary condition (3b) is considered in Section 3.

\section{Blow-Up Time for Dirichlet Boundary Condition}

In this section, we derive a lower bound for $t^{*}$ if the solution $u(x, t) \geq 0$ of (1)-(3a) blows up in finite time $t^{*}$.

Theorem 1. Let $u(x, t)$ be a classical solution of (1)-(3a) with $p+q>\max \{m, s\}$; then a lower bound of the blow-up time for any solution which blows up in $L^{n(p+q-1)}$ norm $(n>\max \{2,(1 /$ $(p+q-1))\})$ is $t^{*} \geq 1 /\left(2 A[\eta(0)]^{2}\right)$, where $A$ is a suitable positive constant given later and $\eta(0)=\int_{\Omega} u_{0}^{n(p+q-1)} d x$.

Proof. Define an auxiliary function of the form

$$
\eta(t)=\int_{\Omega} u^{n(p+q-1)} d x
$$

with

$$
n>\max \left\{2, \frac{1}{p+q-1}\right\} .
$$

Taking the derivative of $\eta(t)$ with respect to $t$ gives

$$
\begin{aligned}
\eta^{\prime}(t)= & n(p+q-1) \int_{\Omega} u^{n(p+q-1)-1} u_{t} d x \\
= & n(p+q-1) \\
& \times \int_{\Omega} u^{n(p+q-1)-1} \\
& \times\left(\Delta u^{m}+u^{p} \int_{\Omega} u^{q} d x-k u^{s}\right) d x \\
= & n(p+q-1) \int_{\Omega} u^{n(p+q-1)-1} \Delta u^{m} d x \\
& +n(p+q-1) \int_{\Omega} u^{n(p+q-1)+p-1} d x \int_{\Omega} u^{q} d x \\
& -n k(p+q-1) \int_{\Omega} u^{n(p+q-1)+s-1} d x \\
= & -m n(p+q-1)[n(p+q-1)-1] \\
& \times \int_{\Omega} u^{n(p+q-1)+m-3}|\nabla u|^{2} d x \\
& +n(p+q-1) \int_{\Omega} u^{n(p+q-1)+p-1} d x \int_{\Omega} u^{q} d x
\end{aligned}
$$

$$
\begin{aligned}
& -n k(p+q-1) \int_{\Omega} u^{n(p+q-1)+s-1} d x \\
= & -\frac{4 m n(p+q-1)[n(p+q-1)-1]}{[n(p+q-1)+m-1]^{2}} \\
& \times \int_{\Omega}\left|\nabla u^{(n(p+q-1)+m-1) / 2}\right|^{2} d x \\
& +n(p+q-1) \int_{\Omega} u^{n(p+q-1)+p-1} d x \int_{\Omega} u^{q} d x \\
& -n k(p+q-1) \int_{\Omega} u^{n(p+q-1)+s-1} d x,
\end{aligned}
$$

where $\nabla$ is the gradient operator.

The application of Hölder inequality to the second term on the right hand side of (6) yields

$$
\begin{aligned}
& \int_{\Omega} u^{q} d x \\
& \leq|\Omega|^{1-(q /(n+1)(p+q-1))} \\
& \quad \times\left(\int_{\Omega} u^{(n+1)(p+q-1)} d x\right)^{q /(n+1)(p+q-1)}, \\
& \int_{\Omega} u^{n(p+q-1)+p-1} d x \\
& \leq|\Omega|^{q /(n+1)(p+q-1)} \\
& \quad \times\left(\int_{\Omega} u^{(n+1)(p+q-1)} d x\right)^{(n(p+q-1)+p-1) /((n+1)(p+q-1))},
\end{aligned}
$$

where $|\Omega|$ denotes the volume of $\Omega$.

By (7), it follows from (6) that

$$
\begin{aligned}
\eta^{\prime}(t) \leq & -\frac{4 m n(p+q-1)[n(p+q-1)-1]}{[n(p+q-1)+m-1]^{2}} \\
& \times \int_{\Omega}\left|\nabla u^{(n(p+q-1)+m-1) / 2}\right|^{2} d x \\
& +n(p+q-1)|\Omega| \int_{\Omega} u^{(n+1)(p+q-1)} d x \\
& -n k(p+q-1) \int_{\Omega} u^{n(p+q-1)+s-1} d x .
\end{aligned}
$$

Let

$$
v=u^{p+q-1}, \quad m_{1}=\frac{m-1}{p+q-1}, \quad \delta=\frac{s-1}{p+q-1}
$$

then

$$
\eta(t)=\int_{\Omega} v^{n} d x
$$


and (8) can be written in the from

$$
\begin{aligned}
\eta^{\prime}(t) \leq & -\frac{4 m n(p+q-1)[n(p+q-1)-1]}{[n(p+q-1)+m-1]^{2}} \\
& \times \int_{\Omega}\left|\nabla v^{\left(n+m_{1}\right) / 2}\right|^{2} d x+n(p+q-1)|\Omega| \\
& \times \int_{\Omega} v^{(n+1)} d x-n k(p+q-1) \int_{\Omega} v^{n+\delta} d x
\end{aligned}
$$

Now we seek a bound for $\int_{\Omega} v^{n+1} d x$ in terms of $\eta$ and the first and third terms on the right in (11). First, the application of Hölder inequality yields

$$
\begin{aligned}
\int_{\Omega} v^{n+1} d x & \\
\leq & \left(\int_{\Omega} v^{n+\delta} d x\right)^{\left(2 n+3 m_{1}-4\right) /\left(2 n+3 m_{1}-4 \delta\right)} \\
& \times\left(\int_{\Omega} v^{\left(6 n+3 m_{1}\right) / 4} d x\right)^{(4-4 \delta) /\left(2 n+3 m_{1}-4 \delta\right)}
\end{aligned}
$$

Using the following Sobolev type inequality (see [12]):

$$
\left(\int_{\Omega}|\emptyset|^{\beta} d x\right)^{1 / \beta} \leq c\left(\int_{\Omega}|\nabla \emptyset|^{\gamma} d x\right)^{1 / \gamma},
$$

with $\beta=6, \gamma=2$, and $c=4^{1 / 3} 3^{-1 / 2} \pi^{-2 / 3}$, we obtain

$$
\begin{aligned}
& \int_{\Omega} v^{n+1} d x \\
& \leq\left(\int_{\Omega} v^{n+\delta} d x\right)^{\left(2 n+3 m_{1}-4\right) /\left(2 n+3 m_{1}-4 \delta\right)} \\
& \times\left[c^{3 / 2}\left(\int_{\Omega} v^{n} d x \int_{\Omega}\left|\nabla v^{\left(n+m_{1}\right) / 2}\right|^{2} d x\right)^{3 / 4}\right]^{(4-4 \delta) /\left(2 n+3 m_{1}-4 \delta\right)}
\end{aligned}
$$

Then for some positive constant $\mu_{1}$ to be determined it follows that

$$
\begin{aligned}
\int_{\Omega} v^{n+1} d x \\
\leq c^{6(1-\delta) /\left(2 n+3 m_{1}-4 \delta\right)} \\
\quad \times\left(\mu_{1}^{4(1-\delta) /\left(2 n+3 m_{1}-4\right)} \int_{\Omega} v^{n+\delta} d x\right)^{\left(2 n+3 m_{1}-4\right) /\left(2 n+3 m_{1}-4 \delta\right)} \\
\quad \times\left[\mu_{1}\left(\int_{\Omega} v^{n} d x \int_{\Omega}\left|\nabla v^{\left(n+m_{1}\right) / 2}\right|^{2} d x\right)^{3 / 4}\right]^{(4-4 \delta) /\left(2 n+3 m_{1}-4 \delta\right)}
\end{aligned}
$$

Next, we use the fundamental inequality

$$
\begin{gathered}
a_{1}^{r_{1}} a_{2}^{r_{2}} \leq r_{1} a_{1}+r_{2} a_{2}, \quad a_{1}, a_{2}>0, r_{1}, r_{2}>0, \\
r_{1}+r_{2}=1,
\end{gathered}
$$

to obtain

$$
\begin{aligned}
\int_{\Omega} v^{n+1} d x & \\
\leq & c^{6(1-\delta) /\left(2 n+3 m_{1}-4 \delta\right)} \\
\times & {\left[\frac{2 n+3 m_{1}-4}{2 n+3 m_{1}-4 \delta} \mu_{1}^{-\left(4(1-\delta) /\left(2 n+3 m_{1}-4\right)\right)}\right.} \\
& \times \int_{\Omega} v^{n+\delta} d x+\frac{4(1-\delta) \mu_{1}}{2 n+3 m_{1}-4 \delta} \\
& \left.\times\left(\int_{\Omega} v^{n} d x \int_{\Omega}\left|\nabla v^{\left(n+m_{1}\right) / 2}\right|^{2} d x\right)^{3 / 4}\right] .
\end{aligned}
$$

Note the fact that, for some positive constant $\mu_{2}$,

$$
\begin{aligned}
& {\left[\left(\int_{\Omega} v^{n} d x\right)^{3}\right]^{1 / 4}\left(\int_{\Omega}\left|\nabla v^{\left(n+m_{1}\right) / 2}\right|^{2} d x\right)^{3 / 4}} \\
& \quad \leq \frac{1}{4 \mu_{2}{ }^{3}}\left(\int_{\Omega} v^{n} d x\right)^{3}+\frac{3 \mu_{2}}{4} \int_{\Omega}\left|\nabla v^{\left(n+m_{1}\right) / 2}\right|^{2} d x
\end{aligned}
$$

Substituting inequality (18) into (17) gives

$$
\begin{aligned}
\int_{\Omega} v^{n+1} d x \leq & c^{6(1-\delta) /\left(2 n+3 m_{1}-4 \delta\right)} \\
& \times\left\{\frac{2 n+3 m_{1}-4}{2 n+3 m_{1}-4 \delta} \mu_{1}^{-\left(4(1-\delta) /\left(2 n+3 m_{1}-4 \delta\right)\right)}\right. \\
& \times \int_{\Omega} v^{n+\delta} d x+\frac{4(1-\delta) \mu_{1}}{2 n+3 m_{1}-4 \delta} \\
& \times\left[\frac{1}{4 \mu_{2}{ }^{3}}\left(\int_{\Omega} v^{n} d x\right)^{3}\right. \\
& \left.\left.+\frac{3 \mu_{2}}{4} \int_{\Omega}\left|\nabla v^{\left(n+m_{1}\right) / 2}\right|^{2} d x\right]\right\}
\end{aligned}
$$

Then, by applying inequality (19), it follows from (11) that

$$
\begin{aligned}
\eta^{\prime}(t) \leq & \left\{3 \mu_{2} c^{6(1-\delta) /\left(2 n+3 m_{1}-4 \delta\right)}\right. \\
& \times \frac{(1-\delta) \mu_{1} n(p+q-1)|\Omega|}{2 n+3 m_{1}-4 \delta} \\
& \left.\quad-\frac{4 m n(p+q-1)[n(p+q-1)-1]}{[n(p+q-1)+m-1]^{2}}\right\} \\
& \times \int_{\Omega}\left|\nabla v^{\left(n+m_{1}\right) / 2}\right|^{2} d x+c^{6(1-\delta) /\left(2 n+3 m_{1}-4 \delta\right)} \\
& \times \frac{(1-\delta) \mu_{1} n(p+q-1)|\Omega|}{\left(2 n+3 m_{1}-4 \delta\right) \mu_{2}{ }^{3}}\left(\int_{\Omega} v^{n} d x\right)^{3}
\end{aligned}
$$




$$
\begin{array}{r}
+\left[c^{6(1-\delta) /\left(2 n+3 m_{1}-4 \delta\right)} \mu_{1}^{-4(1-\delta) /\left(2 n+3 m_{1}-4\right)}\right. \\
\times \frac{\left(2 n+3 m_{1}-4\right) n(p+q-1)|\Omega|}{2 n+3 m_{1}-4 \delta} \\
-n k(p+q-1)] \int_{\Omega} v^{n+\delta} d x
\end{array}
$$

We next choose $\mu_{1}$ to make the coefficient of $\int_{\Omega} v^{n+\delta} d x$ vanish and then choose $\mu_{2}$ to make the coefficient of $\int_{\Omega}\left|\nabla v^{\left(n+m_{1}\right) / 2}\right|^{2} d x$ vanish. It follows that

$$
\eta^{\prime}(t) \leq A[\eta(t)]^{3}
$$

with

$$
A=c^{6(1-\delta) /\left(2 n+3 m_{1}-4 \delta\right)} \frac{\mu_{1} n|\Omega|(1-\delta)(p+q-1)}{\left(2 n+3 m_{1}-4 \delta\right) \mu_{2}^{3}} .
$$

Integrating inequality (21) from 0 to $t$ gives

$$
\frac{1}{[\eta(0)]^{2}}-\frac{1}{[\eta(t)]^{2}} \leq 2 A t
$$

from which we derive a lower bound for $t^{*}$ :

$$
t^{*} \geq \frac{1}{2 A[\eta(0)]^{2}} \text {. }
$$

This completes the proof of Theorem 1 .

\section{Blow-Up Time for Neumann Boundary Condition}

In this final section, we discuss a lower bound for $t^{*}$ if the solution $u(x, t)$ of (1), (2), and (3b) is blow-up in finite time $t^{*}$.

Theorem 2. Let $u(x, t)$ be a classical solution of (1), (2), and (3b) with $p+q>\max \{m, s\}$; then a lower bound of the blow-up time for any solution which blows up in $L^{n(p+q-1)}$ norm is $t^{*} \geq$ $\int_{\eta(0)}^{\infty}\left(d \xi /\left(K_{2} \xi^{(3(n+1)) /\left(n+4-3 m_{1}\right)}+K_{3} \xi^{3}\right)\right)$, where $K_{2}$ and $K_{3}$ are suitable positive constants given later, respectively, and $\eta(0)=$ $\int_{\Omega} u_{0}^{n(p+q-1)} d x$

Proof. We estimate $\int_{\Omega} v^{\left(6 n+3 m_{1}\right) / 4} d x$ in inequality (14). In a similar way to the process of the derivation of (3.3) in [10], we have

$$
\begin{aligned}
\int_{\Omega} v^{3 / 2\left(\left(2 n+m_{1}\right) / 2\right)} d x & \\
\leq \frac{1}{3^{3 / 4}}[ & \frac{3}{2 \rho_{0}} \int_{\Omega} v^{\left(2 n+m_{1}\right) / 2} d x \\
& +\frac{\left(2 n+m_{1}\right)\left(d+\rho_{0}\right)}{4 \rho_{0}}\left(\int_{\Omega} v^{n} d x\right)^{1 / 2} \\
& \left.\times\left(\int_{\Omega}\left|\nabla v^{\left(n+m_{1}\right) / 2}\right|^{2}\right)^{1 / 2}\right]^{3 / 2}
\end{aligned}
$$

where $\rho_{0}=\min _{\partial \Omega} x_{i} v_{i}, d^{2}=\max _{\bar{\Omega}} x_{i} x_{i}, i=1,2,3$, and $v_{i}$ is the $i$ th component of the unit outer normal vector $v$ on $\partial \Omega$. By virtue of Hölder inequality, we get

$$
\begin{aligned}
\int_{\Omega} v^{\left(2 n+m_{1}\right) / 2} d x \leq & \left(\int_{\Omega} v^{n} d x\right)^{1 / 2}\left(\int_{\Omega} v^{n+m_{1}} d x\right)^{1 / 2} \\
\leq & \left(|\Omega|^{\left(1-m_{1}\right) /(n+1)}\left(\int_{\Omega} v^{n+1} d x\right)^{\left(n+m_{1}\right) /(n+1)}\right)^{1 / 2} \\
& \times\left(\int_{\Omega} v^{n} d x\right)^{1 / 2} .
\end{aligned}
$$

Substituting inequality (26) into (25) yields

$$
\begin{aligned}
\int_{\Omega} v^{3 / 2\left(\left(2 n+m_{1}\right) / 2\right)} d x & \\
\leq \frac{1}{3^{3 / 4}}[ & \frac{3}{2 \rho_{0}}\left(|\Omega|^{\left(1-m_{1}\right) /(n+1)}\left(\int_{\Omega} v^{n+1} d x\right)^{\left(n+m_{1}\right) /(n+1)}\right)^{1 / 2} \\
& \times\left(\int_{\Omega} v^{n} d x\right)^{1 / 2} \\
& +\frac{\left(2 n+m_{1}\right)\left(d+\rho_{0}\right)}{4 \rho_{0}}\left(\int_{\Omega} v^{n} d x\right)^{1 / 2} \\
& \left.\times\left(\int_{\Omega}\left|\nabla v^{\left(n+m_{1}\right) / 2}\right|^{2} d x\right)^{1 / 2}\right]^{3 / 2}
\end{aligned}
$$

Applying the following inequality:

$$
\left(a_{1}+a_{2}\right)^{s} \leq 2^{s}\left(a_{1}^{s}+a_{2}^{s}\right), \quad a_{1}, a_{2}>0, s>1,
$$

we conclude that

$$
\begin{aligned}
\int_{\Omega} v^{3 / 2\left(\left(2 n+m_{1}\right) / 2\right)} d x \\
\leq \frac{2^{3 / 2}}{3^{3 / 4}}\left(\frac{3}{2 \rho_{0}}\right)^{3 / 2}|\Omega|^{3\left(1-m_{1}\right) / 4(n+1)} \\
\quad \times\left(\int_{\Omega} v^{n+1} d x\right)^{3\left(n+m_{1}\right) / 4(n+1)} \\
\quad \times\left(\int_{\Omega} v^{n} d x\right)^{3 / 4}+\frac{2^{3 / 2}}{3^{3 / 4}}\left(\frac{\left(2 n+m_{1}\right)\left(d+\rho_{0}\right)}{4 \rho_{0}}\right)^{3 / 2} \\
\quad \times\left(\int_{\Omega} v^{n}\right)^{3 / 4}\left(\int_{\Omega}\left|\nabla v^{\left(n+m_{1}\right) / 2}\right|^{2} d x\right)^{3 / 4} .
\end{aligned}
$$


Applying inequality (16), we obtain

$$
\begin{aligned}
\int_{\Omega} v^{3 / 2\left(\left(2 n+m_{1}\right) / 2\right)} d x \leq & \frac{2^{3 / 2}}{3^{3 / 4}}\left(\frac{3}{2 \rho_{0}}\right)^{3 / 2}|\Omega|^{3\left(1-m_{1}\right) / 4(n+1)} \\
& \times \frac{3\left(n+m_{1}\right)}{4(n+1)} \theta_{1} \int_{\Omega} v^{n+1} d x \\
& +\frac{2^{3 / 2}}{3^{3 / 4}}\left(\frac{3}{2 \rho_{0}}\right)^{3 / 2}|\Omega|^{3\left(1-m_{1}\right) / 4(n+1)} \\
& \times \frac{n+4-3 m_{1}}{4(n+1)} \theta_{1}^{-3\left(n+m_{1}\right) /\left(n+4-3 m_{1}\right)} \\
& \times\left(\int_{\Omega} v^{n} d x\right)^{3(n+1) /\left(n+4-3 m_{1}\right)}+\frac{2^{3 / 2}}{4 \times 3^{3 / 4}} \\
& \times\left(\frac{\left(2 n+m_{1}\right)\left(d+\rho_{0}\right)}{4 \rho_{0}}\right)^{3 / 2} \\
& \times \theta_{2}^{-3}\left(\int_{\Omega} v^{n} d x\right)^{3} \\
& +\frac{3 \times 2^{3 / 2}}{4 \times 3^{3 / 4}}\left(\frac{\left(2 n+m_{1}\right)\left(d+\rho_{0}\right)}{4 \rho_{0}}\right)^{3 / 2}\left|\nabla v^{\left(n+m_{1}\right) / 2}\right|^{2} d x, \\
& +\theta_{2}(30)
\end{aligned}
$$

where $\theta_{1}$ and $\theta_{2}$ are arbitrary positive constants.

Recalling (12) and applying inequality (16) again, for a suitable constant $\mu_{3}$, we obtain

$$
\begin{aligned}
\int_{\Omega} v^{n+1} d x \leq & \frac{2 n+3 m_{1}-4}{2 n+3 m_{1}-4 \delta} \mu_{3}^{-\left(4(1-\delta) /\left(2 n+3 m_{1}-4\right)\right)} \\
& \times \int_{\Omega} v^{n+\delta} d x+\frac{4-4 \delta}{2 n+3 m_{1}-4 \delta} \mu_{3} \\
& \times \int_{\Omega} v^{\left(6 n+3 m_{1}\right) / 4} d x
\end{aligned}
$$

By applying (30), it follows from (31) that

$$
\begin{aligned}
\int_{\Omega} v^{n+1} d x \leq & \frac{2 n+3 m_{1}-4}{2 n+3 m_{1}-4 \delta} \mu_{3}^{-\left(4(1-\delta) /\left(2 n+3 m_{1}-4\right)\right)} \\
& \times \int_{\Omega} v^{n+\delta} d x+\frac{1-\delta}{2 n+3 m_{1}-4 \delta} \frac{2^{3 / 2}}{3^{3 / 4}}\left(\frac{3}{2 \rho_{0}}\right)^{3 / 2} \\
& \times|\Omega|^{3\left(1-m_{1}\right) / 4(n+1)} \frac{3\left(n+m_{1}\right)}{n+1} \theta_{1} \mu_{3} \int_{\Omega} v^{n+1} d x \\
& +\frac{1-\delta}{2 n+3 m_{1}-4 \delta} \frac{2^{3 / 2}}{3^{3 / 4}}\left(\frac{3}{2 \rho_{0}}\right)^{3 / 2}|\Omega|^{3\left(1-m_{1}\right) / 4(n+1)} \\
& \times \frac{n+4-3 m_{1}}{n+1} \theta_{1}^{-3\left(n+m_{1}\right) /\left(n+4-3 m_{1}\right)}
\end{aligned}
$$

$$
\begin{aligned}
& \times \mu_{3}\left(\int_{\Omega} v^{n} d x\right)^{3(n+1) /\left(n+4-3 m_{1}\right)} \\
& +\frac{1-\delta}{2 n+3 m_{1}-4 \delta} \frac{2^{3 / 2}}{3^{3 / 4}}\left(\frac{\left(2 n+m_{1}\right)\left(d+\rho_{0}\right)}{4 \rho_{0}}\right)^{3 / 2} \\
& \times \mu_{3} \theta_{2}^{-3}\left(\int_{\Omega} v^{n} d x\right)^{3}+\frac{3(1-\delta)}{2 n+3 m_{1}-4 \delta} \frac{2^{3 / 2}}{3^{3 / 4}} \\
& \times\left(\frac{\left(2 n+m_{1}\right)\left(d+\rho_{0}\right)}{4 \rho_{0}}\right)^{3 / 2} \\
& \times \mu_{3} \theta_{2} \int_{\Omega}\left|\nabla v^{\left(n+m_{1}\right) / 2}\right|^{2} d x .
\end{aligned}
$$

Taking

$$
\begin{aligned}
K_{0}= & 1-\frac{1-\delta}{2 n+3 m_{1}-4 \delta} \frac{2^{3 / 2}}{3^{3 / 4}}\left(\frac{3}{2 \rho_{0}}\right)^{3 / 2} \\
& \times|\Omega|^{3\left(1-m_{1}\right) / 4(n+1)} \frac{3\left(n+m_{1}\right)}{n+1} \theta_{1} \mu_{3}>0,
\end{aligned}
$$

then combining (32) with (11) gives

$$
\begin{aligned}
\eta^{\prime}(t) \leq & K_{1} \int_{\Omega}\left|\nabla v^{\left(n+m_{1}\right) / 2}\right|^{2} d x \\
& +K_{2}\left(\int_{\Omega} v^{n} d x\right)^{3(n+1) /\left(n+4-3 m_{1}\right)} \\
& +K_{3}\left(\int_{\Omega} v^{n} d x\right)^{3}+K_{4} \int_{\Omega} v^{n+\delta} d x
\end{aligned}
$$

where

$$
\begin{aligned}
K_{1}= & \frac{1}{K_{0}} \frac{3(1-\delta)}{2 n+3 m_{1}-4 \delta} \frac{2^{3 / 2}}{3^{3 / 4}} \\
& \times\left(\frac{\left(2 n+m_{1}\right)\left(d+\rho_{0}\right)}{4 \rho_{0}}\right)^{3 / 2} \\
& \times \mu_{3} \theta_{2} n(p+q-1)|\Omega| \\
& -\frac{4 m n(p+q-1)[n(p+q-1)-1]}{[n(p+q-1)+m-1]^{2}}, \\
K_{2}= & \frac{1}{K_{0}} \frac{3(1-\delta)}{2 n+3 m_{1}-4 \delta} \frac{2^{3 / 2}}{3^{3 / 4}}\left(\frac{3}{2 \rho_{0}}\right)^{3 / 2} \\
& \times|\Omega|^{1+\left(3\left(1-m_{1}\right) / 4(n+1)\right)} \frac{n+4-3 m_{1}}{n+1} \\
& \times \theta_{1}^{-3\left(n+m_{1}\right) /\left(n+4-3 m_{1}\right)} \mu_{3} n(p+q-1),
\end{aligned}
$$




$$
\begin{aligned}
K_{3}= & \frac{1}{K_{0}} \frac{1-\delta}{2 n+3 m_{1}-4 \delta} \frac{2^{3 / 2}}{3^{3 / 4}}\left(\frac{\left(2 n+m_{1}\right)\left(d+\rho_{0}\right)}{4 \rho_{0}}\right)^{3 / 2} \\
& \times \mu_{3} \theta_{2}^{-3} n(p+q-1)|\Omega|, \\
K_{4}= & \frac{1}{K_{0}} \frac{2 n+3 m_{1}-4}{2 n+3 m_{1}-4 \delta} \mu_{3}^{-\left(4(1-\delta) /\left(2 n+3 m_{1}-4\right)\right)} \\
& \times n(p+q-1)|\Omega|-n k(p+q-1) .
\end{aligned}
$$

We can make $K_{1}$ and $K_{4}$ vanish by taking suitable $\mu_{3}, \theta_{1}$, and $\theta_{2}$; then we have

$$
\eta^{\prime}(t) \leq K_{2} \eta^{3(n+1) /\left(n+4-3 m_{1}\right)}+K_{3} \eta^{3} .
$$

Integrating inequality above from 0 to $t$ gives

$$
t \geq \int_{\eta(0)}^{\eta(t)} \frac{d \xi}{K_{2} \xi^{3(n+1) /\left(n+4-3 m_{1}\right)}+K_{3} \xi^{3}},
$$

from which we derive a lower bound for $t<t^{*}$; namely,

$$
t^{*} \geq \int_{\eta(0)}^{\infty} \frac{d \xi}{k_{2} \xi^{3(n+1) /\left(n+4-3 m_{1}\right)}+K_{3} \xi^{3}} .
$$

This completes the proof of Theorem 2 .

\section{Conflict of Interests}

The authors declare that they have no competing interests.

\section{Authors' Contribution}

All authors contributed equally to the paper and read and approved the final paper.

\section{Acknowledgments}

This work is supported by the Natural Science Foundation of Shandong Province of China (ZR2012AM018) and the Fundamental Research Funds for the Central Universities (no. 201362032). The authors would like to deeply thank all the reviewers for their insightful and constructive comments.

\section{References}

[1] J. Bebernes and D. Eberly, Mathematical Problems from Combustion Theory, Springer, New York, NY, USA, 1989.

[2] C. V. Pao, Nonlinear Parabolic and Elliptic Equations, Plenum Press, New York, NY, USA, 1992.

[3] J. Furter and M. Grinfeld, "Local versus non-local interactions in population dynamics," Journal of Mathematical Biology, vol. 27, no. 1, pp. 65-80, 1989.

[4] H. A. Levine, "Nonexistence of global weak solutions to some properly and improperly posed problems of mathematical physics: the method of unbounded fourier coefficients," Mathematische Annalen, vol. 214, no. 3, pp. 205-220, 1975.

[5] X. Gao, J. Ding, and B.-Z. Guo, "Blow-up and global solutions for quasilinear parabolic equations with Neumann boundary conditions," Applicable Analysis, vol. 88, no. 2, pp. 183-191, 2009.
[6] J. Wang, Z. Wang, and J. Yin, "A class of degenerate diffusion equations with mixed boundary conditions," Journal of Mathematical Analysis and Applications, vol. 298, no. 2, pp. 589-603, 2004.

[7] L. E. Payne and P. W. Schaefer, "Lower bounds for blow-up time in parabolic problems under Dirichlet conditions," Journal of Mathematical Analysis and Applications, vol. 328, no. 2, pp. 1196-1205, 2007.

[8] L. E. Payne and J. C. Song, "Lower bounds for blow-up time in a nonlinear parabolic problem," Journal of Mathematical Analysis and Applications, vol. 354, no. 1, pp. 394-396, 2009.

[9] J. C. Song, "Lower bounds for the blow-up time in a non-local reaction-diffusion problem," Applied Mathematics Letters, vol. 24, no. 5, pp. 793-796, 2011.

[10] D. Liu, C. Mu, and Q. Xin, "Lower bounds estimate for the blowup time of a nonlinear nonlocal porous medium equation," Acta Mathematica Scientia B, vol. 32, no. 3, pp. 1206-1212, 2012.

[11] Z. B. Fang, J. Zhang, and S.-C. Yi, "Roles of weight functions to a nonlocal porous medium equation with inner absorption and nonlocal boundary condition," Abstract and Applied Analysis, vol. 2012, Article ID 326527, 16 pages, 2012.

[12] G. Talenti, "Best constant in Sobolev inequality," Annali di Matematica Pura ed Applicata, vol. 110, no. 1, pp. 353-372, 1976. 


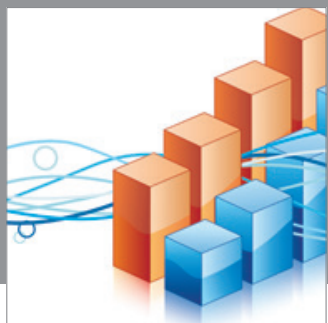

Advances in

Operations Research

mansans

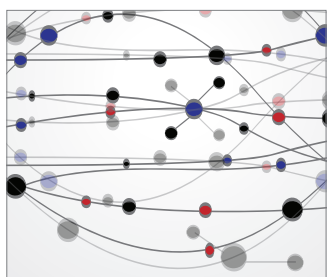

The Scientific World Journal
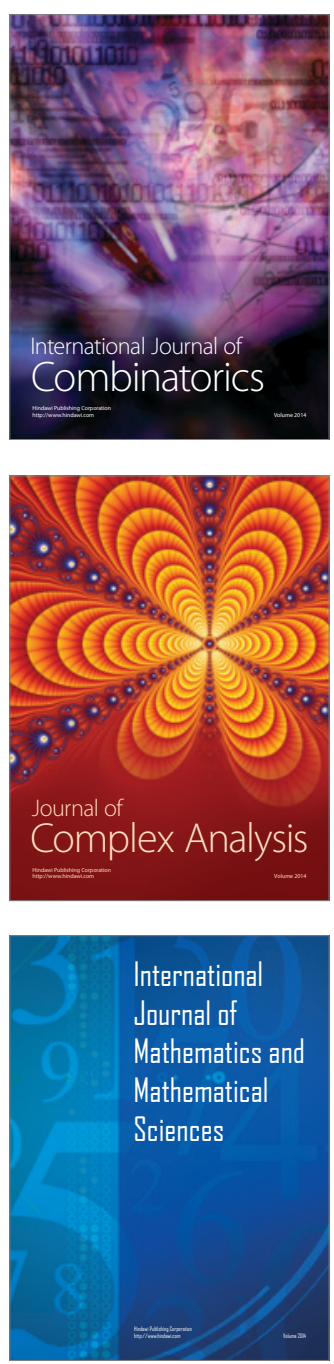
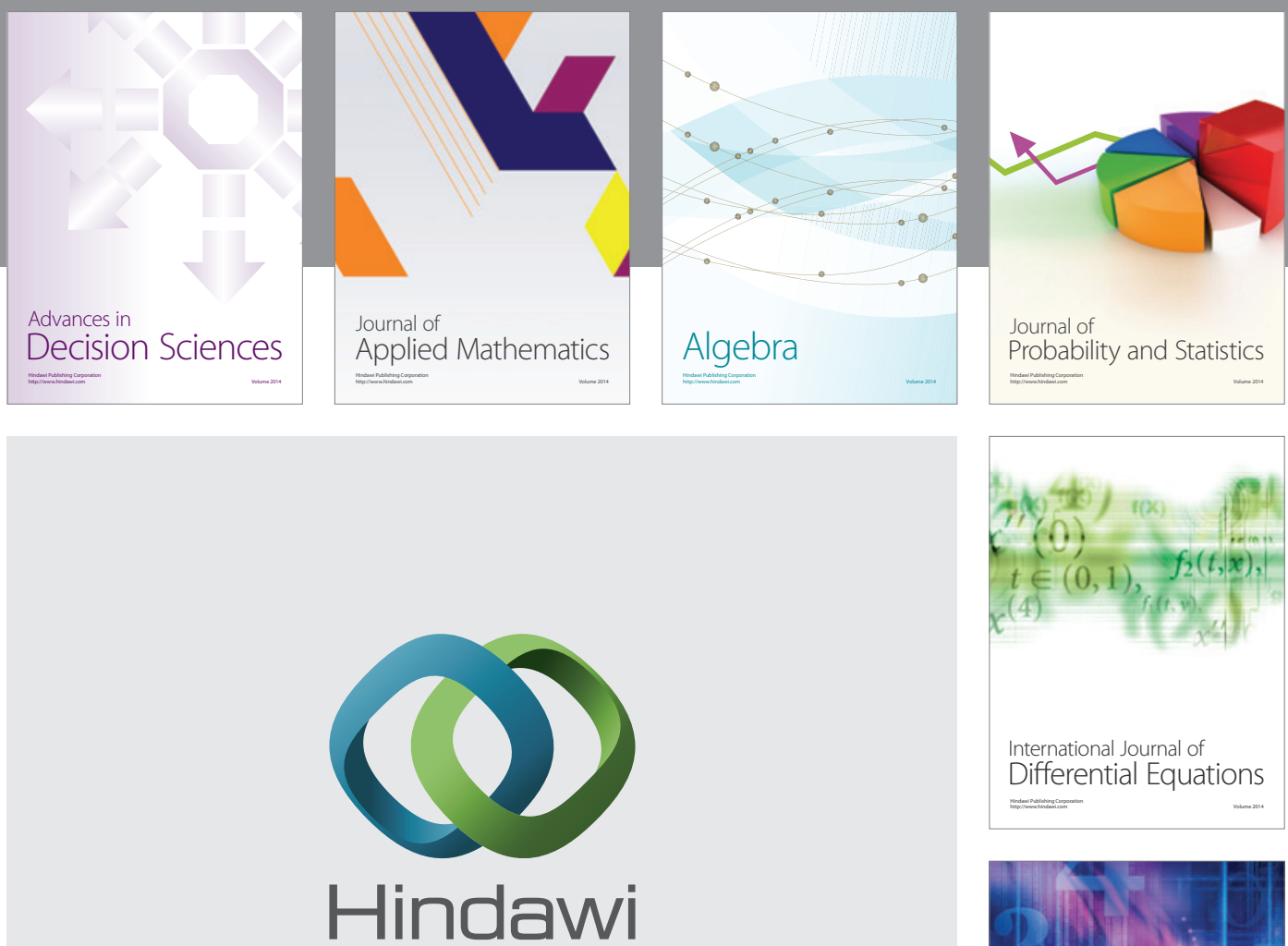

Submit your manuscripts at http://www.hindawi.com
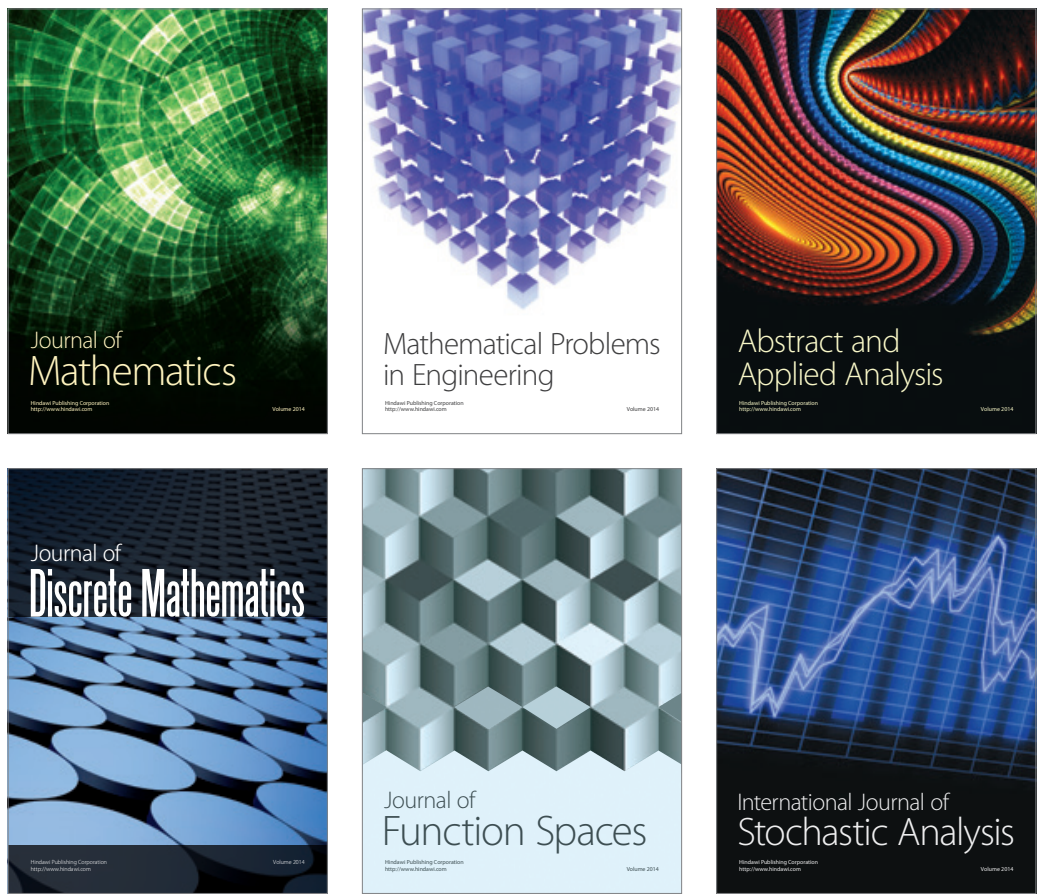

Journal of

Function Spaces

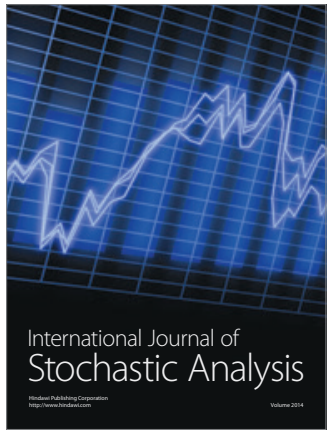

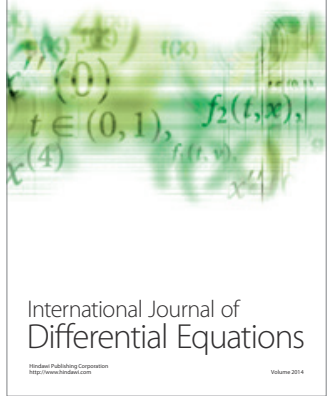
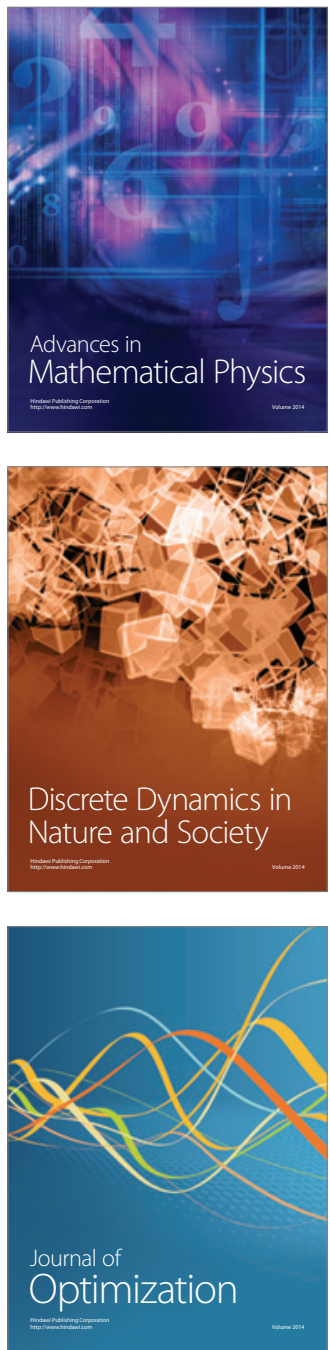